\title{
BIFURCATION ANALYSIS OF NONSTANDARD FINITE DIFFERENCE METHOD FOR PHYSIOLOGICAL CONTROL SYSTEM
}

\author{
JIEYI YAO, QI WANG* \\ School of Mathematics and Statistics, Guangdong University of Technology, Guangzhou 510006, China \\ *Corresponding author: bmwzwq@126.com \\ Received Dec. 5, 2021
}

\begin{abstract}
AвSTRACT. This paper focuses on the bifurcation behavior of physiological control system by the nonstandard finite difference method. By using the Hopf bifurcation theory of discrete system, we prove that a series of Hopf bifurcations appear at the positive equilibrium point with the increase of time delay. At the same time, the existence of local Hopf bifurcations is investigated. Moreover, the direction of the Hopf bifurcation and the stability of bifurcating periodic solutions are verified. Finally, some numerical examples are given to illustrate the theoretical results. 2010 Mathematics Subject Classification. 65P30; 65L12.
\end{abstract}

Key words and phrases. physiological control system; nonstandard finite difference method; Hopf bifurcation; stability.

\section{InTRODUCTION}

In recent years, a great deal of work has been done to the dynamic behavior of delay differential equations (DDEs). Some properties have received widespread attention, such as stability, oscillations and periodicity [1-4]. Since the bifurcation problems in the mathematical models of ecology, biology, neural networks and the spread of some infectious diseases in human beings reflect many realistic dynamic phenomena, so the bifurcation theory of DDEs is becoming more and more popular to the research of qualitative theory of differential equations [5-8].

DOI: 10.28924/APJM/9-3

@2022 Asia Pacific Journal of Mathematics 
Considering the need of scientific computation and real-time simulation, more and more scholars pay attention to the behaviors of discrete system corresponding to the original differential equations. As to the numerical bifurcations of DDEs, some authors applied many classical numerical methods e.g. Runge-Kutta methods, the trapezoidal formula and linear multi-step methods to simulate the Hopf bifurcations [9-12]. For more information on this aspect, the interested readers can refer to the literatures [13-15].

In this paper, we consider a physiological control system which is described by the following nonlinear DDE

$$
\dot{p}(t)=\gamma-\frac{\beta v_{m} p(t) p^{n}(t-\tau)}{\theta^{n}+p^{n}(t-\tau)}, \quad t \geq 0
$$

where $p(t)$ stands for carbon dioxide concentration in arterial, $\gamma$ is the carbon dioxide production rate, $\beta$ is a positive constant, $v_{m}$ is the maximum flow of carbon dioxide, $\tau$ is the time delay between oxidation in the blood of the lungs to chemical reactions in the brainstem, $\theta$ and $n$ are positive parameters obtained by observing different experimenters. This model was first proposed by Mackey and Glass [16], and its solution is similar to the pulmonary ventilation envelope of pathological respiration, which is called Cheyne Stokes Breathing (CSB). CSB is characterized by periodic increasing or decreasing pattern of respiration and alternation of central apnea with cycle length larger than 40 seconds (the most common is 45-60 seconds).

Some scholars have focused on many qualitative properties of (1) such as oscillation, global attractivity, stability and chaos (see [16-19]). Actually, from the viewpoint of control, the discretization form is the most appropriate and realistic to represent the dynamic carbon dioxide concentration in the artery. The reason is that the examination of patients, cardiopulmonary exercise test and the dosage of drugs used in treatment are prescribed as a discrete process. Ding and Su [20] studied the dynamics of solutions of (1) by the midpoint formula. As is known to all, one dynamic consistent numerical method can reproduce the dynamic behavior of differential equations accurately [21]. Specifically, a discrete-time model is said to be dynamically consistent with its continuous counterpart if it demonstrates the similar dynamical behavior, such as boundedness, persistence, stability, chaos and bifurcation [22]. Although the midpoint formula could preserve the local stability and the Hopf bifurcation of some DDEs for any step size, it is implicit method whose computation is a bit large. Thus, different from [20], in this paper, we use another effective and potential method: nonstandard finite difference method (NSFDM). 
Nowadays, the NSFDM have received considerable attention due to the improvement in their efficient computation and potential advantages, such as preserving some essential properties of ordinary and partial differential equations [23]. The basic rules of the NSFDM have been introduced by Mickens [24-27] to solve linear and nonlinear differential equations. This technique is universally known to maintain positivity, boundedness, fixed points and stability of nonlinear systems $[28,29]$. It is also showed that the NSFDM has better convergence than Euler method and Runge-Kutta methods [24,28].

The main work of this article is propose a NSFDM to discretize (1) and analyze the corresponding difference scheme by using the Hopf bifurcation theory of discrete system. We show that the NSFDM can preserve the dynamic properties include the existence and direction of the Hopf bifurcations, the local stability of equilibrium point and the stability of the bifurcating periodic solutions.

The rest of this work is summarized as follows. In Section 2, the stability of the positive equilibrium and the existence of the local Hopf bifurcation at the equilibrium point are studied. In Section 3, the direction and stability of bifurcating periodic solutions are discussed. In Section 4, some numerical examples are carried out to support the theoretical result. Finally, Section 5 is devoted to concluding remarks.

\section{Stability of the positive equilibrium AND local Hopf bifurcations}

In this section, we mainly discuss the stability of the equilibrium and the existence of local Hopf bifurcation by considering time delay as bifurcation parameter of the discrete system.

We first make the change of variable $p(t)=\theta x(t)$, then $(1)$ can be rewritten as

$$
\dot{x}(t)=a-b \frac{x(t) x^{n}(t-\tau)}{1+x^{n}(t-\tau)},
$$

where $a=\gamma / \theta, b=\beta v_{m}$. Further, let $u(t)=x(\tau t)$, so (2) can be reformulated as

$$
\dot{u}(t)=a \tau-b \tau \frac{u(t) u^{n}(t-1)}{1+u^{n}(t-1)},
$$

then the positive equilibrium point $u_{*}$ of (3) satisfies

$$
a \tau-b \tau \frac{u_{*}^{n+1}}{1+u_{*}^{n}}=0 .
$$

For the function $f(x)=b x^{n+1}-a x^{n}-a$, we notice that

$$
f^{\prime}(x)=(n+1) b x^{n}-a n x^{n-1} \begin{cases}<0, & 0<x<\frac{a n}{b(n+1)} \\ >0, & x>\frac{a n}{b(n+1)}\end{cases}
$$


and $f(0)=-a<0$, so we know that the positive equilibrium point $u_{*}$ is unique. Meanwhile, (4) implies that

$$
u_{*}>\frac{a}{b}
$$

Let $h=1 / m\left(m \in Z^{+}\right)$be the step size, we use the NSFDM to discrete (3). In NSFDM, the first-order derivative is approximated by $\left(u_{k+1}-u_{k}\right) / \phi(h)$, where $\phi(h)$ is called denominator function, which is the continuous function of $h$ and satisfies the condition $\phi(h)=h+O\left(h^{2}\right)$, $0<\phi(h)<1$, when $h \rightarrow 0$. In this paper, we select the denominator function $\phi(h)=$ $\left(1-e^{-b h \tau}\right) /(b \tau)$, then the numerical discrete system for $(3)$ is as follows

$$
u_{k+1}=u_{k}+\frac{\left(1-e^{-b \tau h}\right) a}{b}-\frac{\left(1-e^{-b \tau h}\right) u_{k} u_{k-m}^{n}}{1+u_{k-m}^{n}} .
$$

Obviously, (7) and (3) share the same positive equilibrium point $u_{*}$. In order to transform the equilibrium point $u_{*}$ of (7) into the origin, set $y_{k}=u_{k}-u_{*}$, then (7) changes into

$$
\begin{aligned}
y_{k+1}= & \left(1-\frac{\left(y_{k-m}+u_{*}\right)^{n}\left(1-e^{-b h \tau}\right)}{1+\left(y_{k-m}+u_{*}\right)^{n}}\right) y_{k}-\frac{\left(y_{k-m}+u_{*}\right)^{n}\left(1-e^{-b h \tau}\right)}{1+\left(y_{k-m}+u_{*}\right)^{n}} u_{*} \\
& +\frac{\left(1-e^{-b h \tau}\right) a}{b} .
\end{aligned}
$$

By introducing a new variable $Y_{k}=\left(y_{k}, y_{k-1}, \cdots, y_{k-m}\right)^{T},(8)$ is equivalently rewritten as

$$
Y_{k+1}=Q\left(Y_{k}, \tau\right)
$$

where $Q=\left(Q_{0}, Q_{1}, \cdots, Q_{m}\right)^{T}$ and

$$
Q_{i}=\left\{\begin{array}{l}
\left(1-\frac{\left(y_{k-m}+u_{*}\right)^{n}\left(1-e^{-b h \tau}\right)}{1+\left(y_{k-m}+u_{*}\right)^{n}}\right) y_{k}-\frac{\left(y_{k-m}+u_{*}\right)^{n}\left(1-e^{-b h \tau}\right)}{1+\left(y_{k-m}+u_{*}\right)^{n}} u_{*}+\frac{\left(1-e^{-b h \tau}\right) a}{b}, i=0, \\
y_{k-i+1}, 1 \leq i \leq m .
\end{array}\right.
$$

The linearization form of (9) at origin is

$$
Y_{k+1}=D Y_{k}
$$

where

$$
D=\left(\begin{array}{cccccc}
d_{m} & 0 & \cdots & 0 & 0 & d_{0} \\
1 & 0 & \cdots & 0 & 0 & 0 \\
0 & 1 & \cdots & 0 & 0 & 0 \\
\vdots & \vdots & \ddots & \vdots & \vdots & \vdots \\
0 & 0 & \cdots & 1 & 0 & 0 \\
0 & 0 & \cdots & 0 & 1 & 0
\end{array}\right)
$$


and

$$
d_{m}=1-\frac{a\left(1-e^{-b h \tau}\right)}{b u_{*}}, d_{0}=-\frac{a n\left(b u_{*}-a\right)\left(1-e^{-b h \tau}\right)}{b^{2} u_{*}^{2}} .
$$

The characteristic equation of the matrix $D$ is

$$
\lambda^{m+1}-\left(1-\frac{a\left(1-e^{-b h \tau}\right)}{b u_{*}}\right) \lambda^{m}+\frac{a n\left(b u_{*}-a\right)\left(1-e^{-b h \tau}\right)}{b^{2} u_{*}^{2}}=0 .
$$

It is known that the equilibrium point is asymptotically stable if and only if all the characteristic roots of the linearization at the equilibrium point stay in the unit circle. The following results are useful to prove the existence of the local Hopf bifurcation at the equilibrium point.

Lemma 1. All the roots of (13) have modulus less than one for sufficiently small $\tau>0$.

Proof: When $\tau=0$, (13) reduces to $\lambda^{m+1}-\lambda^{m}=0$, which has a single root $\lambda=1$ and $m$-fold root zero. When $\tau \neq 0$ and $\tau$ is sufficiently small, we consider the root $\lambda(\tau)$ of (13) such that $\lambda(0)=1$. Since the function $\lambda=\lambda(\tau)$ is continuous and differentiable about $\tau$, so differentiating both sides of (13) with respect to $\tau$, we get

$$
\frac{d \lambda}{d \tau}=-\frac{a h e^{-b h \tau}\left(\lambda^{m} b u_{*}+b n u_{*}-a n\right)}{\lambda^{m-1} u_{*}\left(\lambda b u_{*}(m+1)+a m\left(1-e^{-b h \tau}\right)-b m u_{*}\right)}
$$

and

$$
\frac{d \bar{\lambda}}{d \tau}=-\frac{a h e^{-b h \tau}\left(\bar{\lambda}^{m} b u_{*}+b n u_{*}-a n\right)}{\bar{\lambda}^{m-1} u_{*}\left(\bar{\lambda} b u_{*}(m+1)+a m\left(1-e^{-b h \tau}\right)-b m u_{*}\right)},
$$

combining with (6) we have

$$
\left.\frac{d|\lambda|^{2}}{d \tau}\right|_{\tau=0, \lambda=1}=\left.\left(\lambda \frac{d \bar{\lambda}}{d \tau}+\bar{\lambda} \frac{d \lambda}{d \tau}\right)\right|_{\tau=0, \lambda=1}=-\frac{2 a h\left(b u_{*}+b u_{*} n-a n\right)}{u_{*}^{2} b}<0 .
$$

As a consequence, $\lambda$ can not cross $\lambda=1$ with increasing of $\tau$. That is, all roots of (13) lie in the unit circle for sufficiently small $\tau>0$. The proof is complete.

As is known to all, a Hopf bifurcation occurs when a complex conjugate pair of eigenvalues of $D$ cross the unit circle as $\tau$ varies. To find the bifurcation points, we need to calculate the eigenvalues on the unit circle. So we have the following result.

Lemma 2. If the condition

$$
\text { an }>b(n-1) u_{*}
$$

holds, then the characteristic equation (13) has no root with modulus one for any $\tau>0$. 
Proof: Suppose that the root on the unit circle is $e^{i \omega}, \omega \in(-\pi, \pi]$. Since (13) is a real polynomial equation, we only need to find $\omega \in(0, \pi]$ for $e^{i \omega}$. Inserting $e^{i \omega}$ into (13) and separating the real part and imaginary part we get

$$
\left\{\begin{array}{l}
\cos \omega+\frac{a n\left(b u_{*}-a\right)\left(1-e^{-b h \tau}\right)}{b^{2} u_{*}^{2}} \cos m \omega=1-\frac{a\left(1-e^{-b h \tau}\right)}{b u_{*}}, \\
\sin \omega-\frac{a n\left(b u_{*}-a\right)\left(1-e^{-b h \tau}\right)}{b^{2} u_{*}^{2}} \sin m \omega=0 .
\end{array}\right.
$$

Since $\tau$ is real and \pm 1 are not the roots of (13), from (18) we deduce that

$$
\cos \omega=1+\frac{a^{2}\left(1-e^{-b h \tau}\right)^{2}\left(b u_{*} n+b u_{*}-a n\right)\left(a n+b u_{*}-b u_{*} n\right)}{2 b^{3} u_{*}^{3}\left(b u_{*}-a\left(1-e^{-b h \tau}\right)\right)},
$$

together with (6) and (17), leads to $\cos \omega>1$, which is a contradiction. The proof is finished.

We further know that if (17) holds true, then there exists a $\tau$ such that the characteristic equation (13) has a root $e^{i \omega}(\omega \in(0, \pi])$. From (18), we have

$$
\frac{a n\left(b u_{*}-a\right)\left(1-e^{-b h \tau}\right)}{b^{2} u_{*}^{2}}=\frac{\sin \omega}{\sin m \omega},
$$

then by (6) we know that the left side of (20) is positive. So there exists a real sequence of $\omega_{k}$ which belongs to the interval $(2 k \pi / m,(2 k+1) \pi / m), k=0,1, \cdots,[(m-1) / 2]$, where [.] stands for the greatest integer function. Clearly, there exists a sequence of $\tau_{k}$ satisfying (18) if $\omega=\omega_{k}$.

Let

$$
\text { an }<b(n-1) u_{*},
$$

then the following lemma is naturally given.

Lemma 3. Under the condition (21), let $\lambda_{k}(\tau)=r_{k}(\tau) e^{i \omega_{k}(\tau)}$ be a root of characteristic equation (13) near $\tau=\tau_{k}$ satisfying $r_{k}\left(\tau_{k}\right)=1$ and $\omega_{k}\left(\tau_{k}\right)=\omega_{k}$, then

$$
\left.\frac{d r_{k}^{2}(\tau)}{d \tau}\right|_{\tau=\tau_{k}, \omega=\omega_{k}}>0
$$

Proof: From (13) we get

$$
\lambda^{m}=\frac{a n\left(b u_{*}-a\right)\left(1-e^{-b h \tau}\right)}{b^{2} u_{*}^{2}\left(\lambda-\left(1-\frac{a\left(1-e^{-b h \tau}\right)}{b u_{*}}\right)\right)} .
$$

Also in view of (14) we have

$$
\begin{aligned}
\bar{\lambda} \frac{d \lambda}{d \tau} & =-\frac{\bar{\lambda} a h e^{-b h \tau}\left(\lambda^{m} b u_{*}+b n u_{*}-a n\right)}{\lambda^{m-1} u_{*}\left(\lambda b u_{*}(m+1)+a m\left(1-e^{-b h \tau}\right)-b m u_{*}\right)} \\
& =-\frac{a h e^{-b h \tau}\left(\lambda^{m} b u_{*}+b n u_{*}-a n\right)}{\lambda^{m} u_{*}\left(\lambda b u_{*}(m+1)+a m\left(1-e^{-b h \tau}\right)-b m u_{*}\right)} .
\end{aligned}
$$


Hence, from (6), (21), (23) and (24) we obtain

$$
\begin{aligned}
\left.\frac{d r_{k}^{2}(\tau)}{d \tau}\right|_{\tau=\tau_{k}, \omega=\omega_{k}} & =\left.\left(\lambda \frac{d \bar{\lambda}}{d \tau}+\bar{\lambda} \frac{d \lambda}{d \tau}\right)\right|_{\tau=\tau_{k}, \omega=\omega_{k}}=2 \operatorname{Re}\left(\left.\bar{\lambda} \frac{d \lambda}{d \tau}\right|_{\tau=\tau_{k}, \omega=\omega_{k}}\right) \\
& =2 \operatorname{Re}\left(\frac{u_{*} b^{2} h e^{-b h \tau}(\lambda-1)}{\left(1-e^{-b h \tau}\right)\left(\lambda b u_{*}(m+1)+a m\left(1-e^{-b h \tau}\right)-b m u_{*}\right)}\right) \\
& =\frac{u_{*} b^{2} h e^{-b h \tau_{k}}\left(1-\cos \omega_{k}\right)\left(b u_{*}+2 b m u_{*}-a m\left(1-e^{-b h \tau_{k}}\right)\right)}{\left(1-e^{-b h \tau_{k}}\right) \mid b u_{*}(m+1) e^{i \omega_{k}+a m\left(1-e^{-b h \tau} k\right)-\left.b m u_{*}\right|^{2}}>0 .} .
\end{aligned}
$$

This completes the proof.

Therefore we have the following result.

Lemma 4. ( $i)$ If (17) holds, then for any $\tau>0$, all roots of the characteristic equation (13) are in the unit circle.

(ii) If (21) holds, then the characteristic equation (13) has a pair of simple roots $e^{ \pm i \omega_{k}}$ on the unit circle when $\tau=\tau_{k}(k=0,1,2, \cdots,[(m-1) / 2])$. If $\tau \in\left[0, \tau_{0}\right)$, then the modulus of roots of the characteristic equation (13) are all less than one. If $\tau=\tau_{0}$, then all roots of (13) have modulus less than one except $e^{ \pm i \omega_{k}}$. If $\tau \in\left(\tau_{k}, \tau_{k+1}\right]$, then $(13)$ has $2(k+1)$ roots which with modulus more than one.

Proof: According to Lemmas 2, 3 and Corollary 2.4 in [30] we can get the proof of (i).

If $(21)$ holds, then there is a sequence of $\tau_{k}(k=0,1,2, \cdots,[(m-1) / 2])$, such that (13) has roots $e^{ \pm i \omega_{k}}$ when $\tau=\tau_{k}$ and $\omega=\omega_{k}$, where $\tau_{k}$ and $\omega_{k}$ are given in (22). If $\tau \in\left[0, \tau_{0}\right)$, then by Lemmas 2, 3, the modulus of the roots of (13) are less than one. If $\tau=\tau_{0}$, then all the roots of (13) have modulus less than one except $e^{ \pm i \omega_{k}}$. Moreover, by Rouche theorem [31], the statement on the number of eigenvalues with modulus more than one can be got directly for $\tau \in\left[\tau_{k}, \tau_{k+1}\right)$. The proof is finished.

By virtue of Lemma 4 we have the next theorem for the stability of the zero solution of (7).

Theorem 1. (i) If (17) holds, then $u=u_{*}$ is asymptotically stable for any $\tau \geq 0$.

(ii) If (21) holds, then $u=u_{*}$ is asymptotically stable for $\tau \in\left[0, \tau_{0}\right)$, and unstable for $\tau>\tau_{0}$.

(iii) Under the same condition of (ii), (7) undergoes a Hopf bifurcation at $u_{*}$ when $\tau=\tau_{k}$, for $k=0,1$, $2, \cdots,[(m-1) / 2]$.

\section{Direction AND STABility OF THE Hopf BifURCATION IN DisCRETE SYSTEM}

In this section, by using the similar technique as the centre manifold theorem and normal form theory in [32], we consider the direction of the local Hopf bifurcation and the stability of the bifurcating periodic solution of system (8) when $\tau=\tau_{0}$. 
Let $\tau=\tau_{0}+\mu, \mu \in R$, then $\mu=0$ is a Hopf bifurcation value of (8). Expand (8) at origin gives

$$
\begin{aligned}
y_{k+1} & =\left(1-\frac{\left(y_{k-m}+u_{*}\right)^{n}\left(1-e^{-b h \tau}\right)}{1+\left(y_{k-m}+u_{*}\right)^{n}}\right) y_{k}+\frac{a n\left(a-b u_{*}\right)\left(1-e^{-b h \tau}\right)}{b^{2} u_{*}^{2}} y_{k-m} \\
& +\frac{a n\left(1-e^{-b h \tau}\right)\left(b u_{*}^{n+1}(n-1)\left(a-u_{*} b\right)+2 n a^{2}\right)}{2 b^{3} u_{*}^{n+4}} y_{k-m}^{2} \\
& +\frac{a n\left(1-e^{-b h \tau}\right)}{6 b^{4} u_{*}^{2 n+6}}\left[b^{2} u_{*}^{2 n+2}(n-1)(n-2)\left(a-b u_{*}\right)\right. \\
& \left.+3 a^{2} n\left((n-1) b u_{*}^{n+1}-a\left(u_{*}^{n}(n+1)-n+1\right)\right)\right] y_{k-m}^{3}+O\left(\left|y_{k-m}\right|^{4}\right) .
\end{aligned}
$$

Thus, we can describe (11) as follows

$$
Y_{k+1}=D Y_{k}+\frac{1}{2} B\left(Y_{k}, Y_{k}\right)+\frac{1}{6} C\left(Y_{k}, Y_{k}, Y_{k}\right)+O\left(\left\|Y_{k}\right\|^{4}\right)
$$

where

$$
B\left(Y_{k}, Y_{k}\right)=\left(b_{0}\left(Y_{k}, Y_{k}\right), 0, \cdots, 0\right)^{T}, C\left(Y_{k}, Y_{k}, Y_{k}\right)=\left(c_{0}\left(Y_{k}, Y_{k}, Y_{k}\right), 0, \cdots, 0\right)^{T}
$$

in which

$$
\left\{\begin{array}{l}
b_{0}(\phi, \psi)=\tilde{b} \phi_{m} \psi_{m}, \\
c_{0}(\phi, \psi, \eta)=\tilde{c} \phi_{m} \psi_{m} \eta_{m},
\end{array}\right.
$$

here

$$
\begin{aligned}
\tilde{b}= & \frac{a n\left(1-e^{-b h \tau}\right)\left(b u_{*}^{n+1}(n-1)\left(a-u_{*} b\right)+2 n a^{2}\right)}{b^{3} u_{*}^{n+4}}, \\
\tilde{c}= & \frac{a n\left(1-e^{-b h \tau}\right)}{b^{4} u_{*}^{2 n+6}}\left[b^{2} u_{*}^{2 n+2}(n-1)(n-2)\left(a-b u_{*}\right)\right. \\
& \left.+3 a^{2} n\left((n-1) b u_{*}^{n+1}-a\left(u_{*}^{n}(n+1)-n+1\right)\right)\right] .
\end{aligned}
$$

Let $q=q\left(\tau_{0}\right) \in C^{m+1}$ be an eigenvector of $D$ corresponding to $e^{i \omega_{0}}$, then

$$
D q=e^{i \omega_{0}} q, D \bar{q}=e^{-i \omega_{0}} \bar{q}
$$

Introducing an adjoint eigenvector $q^{*}=q^{*}(\tau) \in C^{m+1}$ with the properties

$$
D^{T} q^{*}=e^{-i \omega_{0}} q^{*}, D^{T} \bar{q}^{*}=e^{i \omega_{0}} \bar{q}^{*}
$$

and satisfying $\left\langle q^{*}, q\right\rangle=\sum_{i=0}^{m} \bar{q}_{i}^{*} q_{i}=1$.

Lemma 5. [33] Define a vector valued function $q: C \rightarrow C^{m+1}$ by

$$
p(\xi)=\left(\xi^{m}, \xi^{m-1}, \cdots, 1\right)^{T},
$$

if $\xi$ is an eigenvalue of $D$, then $D p(\xi)=\xi p(\xi)$. 
By Lemma 5 we have

$$
q=p\left(e^{i \omega_{0}}\right)=\left(e^{i m \omega_{0}}, e^{i(m-1) \omega_{0}}, \cdots, e^{i \omega_{0}}, 1\right)^{T} .
$$

Lemma 6. Suppose $q^{*}=\left(q_{0}^{*}, q_{1}^{*}, \cdots, q_{m}^{*}\right)^{T}$ is the eigenvector of $D^{T}$ corresponding to eigenvalue $e^{-i \omega_{0}}$ and $\left\langle q^{*}, q\right\rangle=1$, then

$$
q^{*}=\bar{K}\left(1, d_{0} e^{i m \omega_{0}}, d_{0} e^{i(m-1) \omega_{0}}, \cdots, d_{0} e^{i 2 \omega_{0}}, d_{0} e^{i \omega_{0}}\right)^{T}
$$

and

$$
K=1 /\left(e^{i m \omega_{0}}+m d_{0} e^{-i \omega_{0}}\right) .
$$

Proof: Assume $q^{*}$ satisfies $D^{T} q^{*}=\bar{z} q^{*}$ with $\bar{z}=e^{-i \omega_{0}}$, then the following identities hold

$$
\left\{\begin{array}{l}
d_{m} q_{0}^{*}+q_{1}^{*}=e^{-i \omega_{0}} q_{0}^{*}, \\
q_{k}^{*}=e^{-i \omega_{0}} q_{k-1}^{*}, \quad k=2,3, \cdots, m, \\
d_{0} q_{0}^{*}=e^{-i \omega_{0}} q_{m}^{*} .
\end{array}\right.
$$

Let $q_{m}^{*}=d_{0} e^{i \omega_{0}} \bar{K}$, then we obtain

$$
q^{*}=\bar{K}\left(1, d_{0} e^{i m \omega_{0}}, d_{0} e^{i(m-1) \omega_{0}}, \cdots, d_{0} e^{i 2 \omega_{0}}, d_{0} e^{i \omega_{0}}\right)^{T} .
$$

Further, form $\left\langle q^{*}, q\right\rangle=1$ and some computations, (33) is hold. So the proof is complete.

Suppose that $a(\lambda)$ is the characteristic polynomial of the matrix $D$ and $\lambda_{0}=e^{i \omega_{0}}$. According to the similar algorithms and computation process in [33], we obtain the expression for the critical coefficient $c_{1}\left(\tau_{0}\right)$ as follows

$$
c_{1}\left(\tau_{0}\right)=\frac{g_{20} g_{11}\left(1-2 \lambda_{0}\right)}{2\left(\lambda_{0}^{2}-\lambda_{0}\right)}+\frac{\left|g_{11}\right|^{2}}{1-\bar{\lambda}_{0}}+\frac{\left|g_{02}\right|^{2}}{2\left(\lambda_{0}^{2}-\bar{\lambda}_{0}\right)}+\frac{g_{21}}{2},
$$

where

$$
\begin{aligned}
& g_{20}=\left\langle q^{*}, B(q, q)\right\rangle, \\
& g_{11}=\left\langle q^{*}, B(q, \bar{q})\right\rangle, \\
& g_{02}=\left\langle q^{*}, B(\bar{q}, \bar{q})\right\rangle, \\
& g_{21}=\left\langle q^{*}, B\left(\bar{q}, \omega_{20}\right)\right\rangle+2\left\langle q^{*}, B\left(q, \omega_{11}\right)\right\rangle+\left\langle q^{*}, C(q, q, \bar{q})\right\rangle,
\end{aligned}
$$

and

$$
\begin{aligned}
& \omega_{20}=\frac{b_{0}(q, q)}{a\left(\lambda_{0}\right)} p\left(\lambda_{0}^{2}\right)-\frac{\left\langle q^{*}, B(q, q)\right\rangle}{\lambda_{0}^{2}-\lambda_{0}} q-\frac{\left\langle\bar{q}^{*}, B(q, q)\right\rangle}{\lambda_{0}^{2}-\bar{\lambda}_{0}} \bar{q}, \\
& \omega_{11}=\frac{b_{0}(q, \bar{q})}{a(1)} p(1)-\frac{\left\langle q^{*}, B(q, \bar{q})\right\rangle}{1-\lambda_{0}} q-\frac{\left\langle\bar{q}^{*}, B(q, \bar{q})\right\rangle}{1-\bar{\lambda}_{0}} \bar{q} .
\end{aligned}
$$


From (28), (31) and Lemma 6, direct calculation produces

$$
\left\{\begin{array}{l}
b_{0}\left(\bar{q}, p\left(e^{i 2 \omega_{0}}\right)\right)=\tilde{b}, \\
b_{0}(q, q)=\tilde{b} \\
b_{0}(q, \bar{q})=\tilde{b} \\
c_{0}(q, q, \bar{q})=\tilde{c}, \\
a\left(e^{i 2 \omega_{0}}\right)=e^{i 2(m+1) \omega_{0}}-d_{m} e^{i 2 m \omega_{0}}-d_{0}, \\
a(1)=1-d_{m}-d_{0}, \\
b_{0}(q, p(1))=\tilde{b} .
\end{array}\right.
$$

By substituting (36) into (35) we deduce that

$$
\begin{aligned}
c_{1}\left(\tau_{0}\right) & =\frac{K}{2}\left(\frac{\tilde{b}^{2}}{a\left(e^{i 2 \omega_{0}}\right)}+\frac{2 \tilde{b}^{2}}{a(1)}+\tilde{c}\right) \\
& =\frac{1}{2\left(e^{i m \omega_{0}}+m d_{0} e^{-i \omega_{0}}\right)}\left(\frac{\tilde{b}^{2}}{e^{i 2(m+1) \omega_{0}}-d_{m} e^{i 2 m \omega_{0}-d_{0}}}+\frac{2 \tilde{b}^{2}}{1-d_{m}-d_{0}}+\tilde{c}\right),
\end{aligned}
$$

where $\tilde{b}, \tilde{c}$ and $K$ are defined in (29), (30) and (33), respectively.

From [34] we have the following lemma.

Lemma 7. Given the map (9) and assume

(i) $\lambda(\tau)=r(\tau) e^{i \omega(\tau)}$, where $r\left(\tau^{*}\right)=1, r^{\prime}\left(\tau^{*}\right) \neq 0$ and $\omega\left(\tau^{*}\right)=\omega^{*}$;

(ii) $e^{i k \omega^{*}} \neq 1$ for $k=1,2,3,4$;

(iii)

$$
\begin{aligned}
& \operatorname{Re}\left[e^{-i \omega^{*}} c_{1}\left(\tau^{*}\right)\right] \\
= & \frac{1}{2}\left(W \tilde{b}^{2}+\frac{\cos (m+1) \omega^{*}+m d_{0}}{1+2 m d_{0} \cos (m+1) \omega^{*}+m^{2} d_{0}^{2}}\left(\frac{2 \tilde{b}^{2}}{1-d_{m}-d_{0}}+\tilde{c}\right)\right) \neq 0,
\end{aligned}
$$

where

$$
W=\frac{A_{1}-A_{2}-A_{3}}{A_{4} A_{5}}
$$

and

$$
\begin{aligned}
& A_{1}=\cos (3 m+3) \omega^{*}, \\
& A_{2}=d_{m}\left(\cos (3 m+1) \omega^{*}+m d_{0} \cos 2 m \omega^{*}\right), \\
& A_{3}=d_{0}\left(\cos (m+1) \omega^{*}-m \cos 2(m+1) \omega^{*}+m d_{0}\right), \\
& A_{4}=1+2 m d_{0} \cos (m+1) \omega^{*}+m^{2} d_{0}^{2}, \\
& A_{5}=1+d_{m}^{2}+d_{0}^{2}-2 d_{m} \cos 2 \omega^{*}-2 d_{0} \cos 2(m+1) \omega^{*}+2 d_{0} d_{m} \cos 2 m \omega^{*},
\end{aligned}
$$

$\tilde{b}$ and $\tilde{c}$ are defined in (29) and (30), respectively. Then an invariant closed curve, topologically equivalent to a circle, for the map (9) exists for $\tau$ in a one side neighborhood of $\tau^{*}$. The radius of the invariant curve grows like $O\left(\sqrt{\left|\tau-\tau^{*}\right|}\right)$. One of the four cases holds:

(i) $r^{\prime}\left(\tau^{*}\right)>0, \operatorname{Re}\left[e^{-i \omega^{*}} c_{1}\left(\tau^{*}\right)\right]<0$. The origin is asymptotically stable for $\tau<\tau^{*}$ and unstable for 
$\tau>\tau^{*}$. An attracting invariant closed curve exists for $\tau>\tau^{*}$;

(ii) $r^{\prime}\left(\tau^{*}\right)>0, \operatorname{Re}\left[e^{-i \omega^{*}} c_{1}\left(\tau^{*}\right)\right]>0$. The origin is asymptotically stable for $\tau<\tau^{*}$ and unstable for $\tau>\tau^{*}$. A repelling invariant closed curve exists for $\tau<\tau^{*}$;

(iii) $r^{\prime}\left(\tau^{*}\right)<0, \operatorname{Re}\left[e^{-i \omega^{*}} c_{1}\left(\tau^{*}\right)\right]<0$. The origin is asymptotically stable for $\tau>\tau^{*}$ and unstable for $\tau<\tau^{*}$. An attracting invariant closed curve exists for $\tau<\tau^{*}$;

(iv) $r^{\prime}\left(\tau^{*}\right)<0, \operatorname{Re}\left[e^{-i \omega^{*}} c_{1}\left(\tau^{*}\right)\right]>0$. The origin is asymptotically stable for $\tau>\tau^{*}$ and unstable for $\tau<\tau^{*}$. A repelling invariant closed curve exists for $\tau>\tau^{*}$.

Recall the discussion in Section 2 we know that $r^{\prime}\left(\tau^{*}\right)>0$. Thus, we have the following result from Lemma 7.

Theorem 2. If (21) holds, then $u=u_{*}$ is asymptotically stable for $\tau \in\left[0, \tau_{0}\right)$, and unstable for $\tau>\tau_{0}$. An attracting (repelling) invariant closed curve exists for $\tau>\tau_{0}$ if $\operatorname{Re}\left[e^{-i \omega_{0}} c_{1}\left(\tau_{0}\right)\right]<0(>0)$.

\section{NumericAl EXPERIMENTS}

In this section, the theoretical results in the previous sections are verified by some numerical examples. Let $a=0.5, b=1, n=4$ in (3) and the initial condition $u(t)=1.1$ for $-\tau \leq t \leq 0$, then we compute that the equilibrium point is $u_{*}=1$ and Inequality (21) holds.

Firstly, in Table 1, we calculate the absolute error (AE) and relative error (RE) of (3) at $t=3$ by explicit Euler method, implicit Euler method and NSFDM with $\tau=0.1$ and different $h$, respectively. It is easy to see from this table that the NSFDM is superior to the explicit Euler method and the implicit Euler method. In Fig. 1, we plot the graphs of the analytic solutions and numerical solutions of (3) by the NSFDM, respectively. From this figure we can see that the analytic solutions and numerical solutions show the same stability and oscillation for every case. Thus, the NSFDM behaves a good preservation manner in stability and oscillation. 
TABLE 1. The errors of three kinds of numerical methods

\begin{tabular}{ccccccccc}
\hline & \multicolumn{2}{c}{ Explicit Euler method } & & \multicolumn{2}{c}{ Implicit Euler method } & & \multicolumn{2}{c}{ NSFDM } \\
\cline { 2 - 3 } & AE & RE & & AE & RE & & AE & RE \\
\hline$h=1 / 2$ & $1.16 \mathrm{E}-03$ & $1.09 \mathrm{E}-03$ & & $1.07 \mathrm{E}-03$ & $1.01 \mathrm{E}-03$ & & $2.80 \mathrm{E}-04$ & $2.64 \mathrm{E}-04$ \\
$h=1 / 4$ & $5.67 \mathrm{E}-04$ & $5.34 \mathrm{E}-04$ & & $5.47 \mathrm{E}-04$ & $5.16 \mathrm{E}-04$ & & $1.39 \mathrm{E}-04$ & $1.31 \mathrm{E}-04$ \\
$h=1 / 8$ & $2.80 \mathrm{E}-04$ & $2.64 \mathrm{E}-04$ & & $2.76 \mathrm{E}-04$ & $2.61 \mathrm{E}-04$ & & $6.88 \mathrm{E}-05$ & $6.48 \mathrm{E}-05$ \\
$h=1 / 16$ & $1.39 \mathrm{E}-04$ & $1.31 \mathrm{E}-04$ & & $1.39 \mathrm{E}-04$ & $1.31 \mathrm{E}-04$ & & $3.40 \mathrm{E}-05$ & $3.21 \mathrm{E}-05$ \\
$h=1 / 32$ & $6.91 \mathrm{E}-05$ & $6.52 \mathrm{E}-05$ & & $7.00 \mathrm{E}-05$ & $6.60 \mathrm{E}-05$ & & $1.67 \mathrm{E}-05$ & $1.57 \mathrm{E}-05$ \\
$h=1 / 64$ & $3.42 \mathrm{E}-05$ & $3.23 \mathrm{E}-05$ & & $3.54 \mathrm{E}-05$ & $3.34 \mathrm{E}-05$ & & $8.03 \mathrm{E}-06$ & $7.57 \mathrm{E}-06$ \\
$h=1 / 128$ & $1.68 \mathrm{E}-05$ & $1.58 \mathrm{E}-05$ & & $1.80 \mathrm{E}-05$ & $1.70 \mathrm{E}-05$ & & $3.70 \mathrm{E}-06$ & $3.49 \mathrm{E}-06$ \\
\hline
\end{tabular}

Next, we illustrate the influence of step size and time delay on the bifurcation value, stability and direction of the Hopf bifurcation by data and figures. In Tables 2 and 3, we compute the bifurcation points, the critical coefficient and the direction of bifurcation at equilibrium point of numerical solutions of (3) with the NSFDM for some different step sizes. Furthermore, the figures of numerical solutions and the related phase diagrams are displayed in Figs. 2-9. From Theorem 1 and Table 2, we conclude that all these numerical solutions should be asymptotically stable for $\tau \in\left[0, \tau_{0}\right)$, unstable for $\tau>\tau_{0}$. This is just what we can see from Figs. 2, 4, 6, 8 . Specifically, in Fig. 2, let $h=1 / 2, \tau=1.7,2.1972$ and 4.5, respectively. It is easy to observe that the change of numerical solutions from stable to unstable is consistent with Theorem 1. We can verify the other three figures in the similar way. Moreover, in view of Theorem 2 and Table 3 , we also obtain that the equilibrium point $u_{*}=1$ is asymptotically stable for $\tau \in\left[0, \tau_{0}\right)$, and unstable for $\tau>\tau_{0}$ and (7) undergoes a Hopf bifurcation at $u_{*}=1$. At the same time, an attracting invariant closed curve exists for $\tau>\tau_{0}$ and $\operatorname{Re}\left[e^{-i \omega_{0}} c_{1}\left(\tau_{0}\right)\right]<0$. This is just what we can see from Figs. 3, 5, 7, 9. More specifically, in the first row of Fig. 3, set $h=1 / 2, \tau=1.7$ and 4.5 , respectively. We can easily see that $u_{*}=1$ is asymptotically stable for $\tau=1.7$ and an attracting invariant closed curve exists for $\tau=4.5$. That is, the bifurcation occurs when $\tau>\tau_{0}=2.1972$ and bifurcating periodic solutions from the equilibrium point are orbitally asymptotically stable, which is consistent with Theorem 2 . We can test the other three figures in a similar fashion.

Finally, from Figs. 3, 5, 7, 9 we also know that the NSFDM preserves the local stability of equilibrium point and the stability of the bifurcating periodic solutions of (3). 
In a word, we can see intuitively that all the numerical phenomena are consistent with the theoretical results.

TABLE 2. The bifurcation points for different step sizes

\begin{tabular}{|c|c|c|c|c|c|}
\hline & $\tau_{0}$ & $\tau_{1}$ & $\tau_{2}$ & $\tau_{3}$ & $\tau_{4}$ \\
\hline$h=1 / 2$ & 2.1972 & & & & \\
\hline$h=1 / 4$ & 1.5288 & 2.4905 & & & \\
\hline$h=1 / 8$ & 1.3468 & 2.4508 & 14.1605 & 14.3958 & \\
\hline$h=1 / 16$ & 1.2736 & 2.4342 & 10.2685 & 11.1239 & 23.6683 \\
\hline
\end{tabular}

TABLE 3. The critical coefficient and directions of bifurcation for different step sizes

\begin{tabular}{ccc}
\hline & $c_{1}\left(\tau_{0}\right)$ & $\operatorname{Re}\left[e^{-i \omega_{0}} c_{1}\left(\tau_{0}\right)\right]$ \\
\hline & & \\
$h=1 / 2$ & $-0.469580-1.138641 \mathrm{i}$ & -1.105322 \\
$h=1 / 4$ & $-0.340576-0.656694 \mathrm{i}$ & -0.519993 \\
$h=1 / 8$ & $-0.186749-0.353175 \mathrm{i}$ & -0.232634 \\
$h=1 / 16$ & $-0.108159-0.176739 \mathrm{i}$ & -0.119847 \\
\hline
\end{tabular}

ACKNOWLEDGEMENTS

This research is supported by the Natural Science Foundation of Guangdong Province (No. 2017A030313031).

\section{REFERENCES}

[1] O. Ocalan, N. Kilic and U.M. Ozkan, Oscillatory behavior for nonlinear delay differential equation with several non-monotone arguments, Comput. Methods Differ. Equ. 8 (2020), 14-27. https: //doi .org/10. 22034/cmde . 2019. 9470.

[2] S.A. Olayemi and B.S. Ogundare, Periodic solutions to certain classes of third order delay differential equations, Tbilisi Math. J. 13 (2020),171-187. https://doi .org/10.32513/tbilisi/1601344907.

[3] J.D. Wei, L.X. Tian, J.B. Zhou, Z.L. Zhen and J. Xu, Existence and asymptotic behavior of traveling wave fronts for a food-limited population model with spatio-temporal delay, Japan J. Ind. Appl. Math. 34 (2017), 305-320. https://doi.org/10.1007/s13160-017-0244-1. 
[4] J.H. Xu, Z.C. Zhang and T. Caraballo, Non-autonomous nonlocal partial differential equations with delay and memory, J. Differ. Equ. 270 (2021), 505-546. https ://doi .org/10.1016/j · jde .2020 .07 . 037.

[5] X.Y. Meng and J.G. Wang, Dynamical analysis of a delayed diffusive predator-prey model with schooling behaviour and Allee effect, J. Biol. Dynam. 14 (2020), 826-848. https://doi .org/10 .1080/17513758 . 2020. 1850892.

[6] H.Y. Shu, W.X. Xu and X.S. Wang, Complex dynamics in a delay differential equation with two delays in tick growth with diapause, J. Differ. Equ. 269 (2020), 10937-10963. https://doi .org/10.1016/j . jde. 2020.07 .029 .

[7] Z. Wang, L. Li, Y.X. Li and Z.S. Cheng, Stability and hopf bifurcation of a three-neuron network with multiple discrete and distributed delays, Neural Process. Lett. 48 (2018), 1481-1502. https://doi .org/10. 1007/s11063-017-9754-8.

[8] C.Y. Xu, Q. Li, T.H. Zhang and S.L. Yuan, Stability and Hopf bifurcation for a delayed diffusive competition model with saturation effect, Math. Biosci. Eng. 17 (2020), 8037-8051. https://doi.org/10.3934/mbe. 2020407.

[9] X.H. Ding, D.J. Fan and M.Z. Liu, Stability and bifurcation of a numerical discretization Mackey-Glass system, Chaos Soliton. Fract. 34 (2007), 383-393. https://doi .org/10.1016/j . chaos . 2006 .03. 053.

[10] M.Z. Liu and Q.B. Wang, Numerical Hopf bifurcation of linear multistep methods for a class of delay differential equations, Appl. Math. Comput. 208 (2009), 462-474. https://doi .org/10.1016/j . amc . 2008. 12.013.

[11] V. Wulf and N.J. Ford, Numerical Hopf bifurcation for a class of delay differential equations, J. Comput. Appl. Math. 115 (2000), 601-616. https://doi .org/10.1016/S0377-0427 (99)00181-8.

[12] L. Zhang, C.J. Zhang and D.M. Zhao, Hopf bifurcation analysis of integro-differential equation with unbounded delay, Appl. Math. Comput. 217 (2011), 4972-4979. https://doi .org/10.1016/j . amc . 2010. 11.046.

[13] D. Breda, O. Diekmann, M. Gyllenberg, F. Scarabel and R. Vermiglio, Pseudospectral discretization of nonlinear delay equations: New prospects for numerical bifurcation analysis, SIAM J. Appl. Dyn. Syst. 15 (2016), 1-23. https://doi.org/10.1137/15M1040931.

[14] Q. Din and K. Haider, Discretization, bifurcation analysis and chaos control for Schnakenberg model, J. Math. Chem. 58 (2020), 1615-1649. https://doi.org/10.1007/s10910-020-01154-x.

[15] X.W. Jiang, X.S. Zhan, Z.H. Guan, X.H. Zhang and L. Yu, Neimark-Sacker bifurcation analysis on a numerical discretization of Gause-type predator-prey model with delay, J. Franklin Inst. 352 (2015), 1-15. https: //doi.org/10.1016/j.jfranklin.2014.09.022.

[16] M.C. Mackey and L. Glass, Oscillation and chaos in physiological control systems, Science. 197 (1977), 287-289. https://doi.org/10.1126/science. 267326.

[17] J.F. Gao, M.H. Song and M.Z. Liu, Oscillation analysis of numerical solutions for nonlinear delay differential equations of population dynamics, Math. Model. Anal. 16 (2011), 365-375. https://doi .org/10. 3846/ 13926292.2011 .601768$. 
[18] K. Gopalsamy, M.R.S. Kulenovic and G. Ladas, Oscillations and global attractivity in respiratory dynamics, Dynam. Stabil. Syst. 4 (1989), 131-139. https://doi .org/10.1080/02681118908806068.

[19] I. Kubiaczyk and S.H. Saker, Oscillation and stability in nonlinear delay differential equations of population dynamics, Math. Comput. Model. 35 (2002), 295-301. https://doi.org/10.1016/S0895-7177(01) $00166-2$.

[20] X.H. Ding and H. Su, Dynamics of a discretization physiological control system, Discrete Dyn. Nat. Soc. 2007 (2007), Article ID 51406. https://doi .org/10.1155/2007/51406.

[21] Q.B. Wang, D.S. Li and M.Z. Liu, Numerical Hopf bifurcation of Runge-Kutta methods for a class of delay differential equations, Chaos Soliton. Fract. 42 (2009), 3087-3099. https://doi.org/10.1016/j. chaos . 2009.04 .008$.

[22] M. Chapwanya, J.M.S. Lubuma and R.E. Mickens, Positivity-preserving nonstandard finite difference schemes for cross-diffusion equations in biosciences, Comput. Math. Appl. 68 (2014), 1071-1082. https: //doi.org/10.1016/j. camwa.2014.04.021.

[23] R. Anguelov, T. Berge, M. Chapwanya, J.K. Djoko, P. Kama, J.M.S. Lubuma and Y. Terefe, Nonstandard finite difference method revisited and application to the Ebola virus disease transmission dynamics, J. Differ. Equ. Appl. 26 (2020), 818-854. https://doi .org/10.1080/10236198.2020.1792892.

[24] R.E. Mickens, Nonstandard Finite Difference Models of Differential Equations, World Scientific, USA (1994).

[25] R.E. Mickens, Nonstandard finite difference schemes for reaction-diffusion equations, Numer. Meth. Part. Differ. Equ. 15 (1999), 201-214. https://doi.org/10.1002/(SICI) 1098-2426(199903) 15:2<201: : AID-NUM5 $>3$. $0 . \mathrm{CO} ; 2-\mathrm{H}$.

[26] R.E. Mickens, Nonstandard finite difference schemes for differential equations, J. Differ. Equ. Appl. 8 (2002), 823-847. https://doi.org/10.1080/1023619021000000807.

[27] R.E. Mickens, A nonstandard finite difference scheme for a Fisher PDE having nonlinear diffusion, Comput. Math. Appl. 45 (2003), 429-436. https : //doi.org/10.1016/S0898-1221 (03)80028-7.

[28] R.E. Mickens, Applications of Nonstandard Finite Difference Schemes. World Scientific, Singapore (2000).

[29] R.E. Mickens and T.M. Washington, A note on a positivity preserving nonstandard finite difference scheme for a modified parabolic reaction-advection-diffusion PDE, J. Differ. Equ. Appl. 26 (2020), 1423-1427. https://doi.org/10.1080/10236198.2020.1841755.

[30] S.G. Ruan and J.J. Wei, On the zeros of transcendental functions with applications to stability of delay differential equations with two delays, Dyn. Contin. Discr. Impulsive Syst. Ser. A: Math. Anal. 10 (2003), 863-874

[31] J. Dieudonne, Foundations of Modern Analysis, Academic Press, USA (1960).

[32] A.K. Yuri, Elements of Applied Bifurcation Theory, Springer-Verlag, USA (1995).

[33] V. Wulf, Numerical Analysis of Delay Differential Equations Undergoing a Hopf Bifurcation, PhD thesis, University of Liverpool, UK (1999).

[34] S. Wiggins, Introduction to Applied Nonlinear Dynamical System and Chaos, Springer-Verlag, USA (1990). 
Appendix
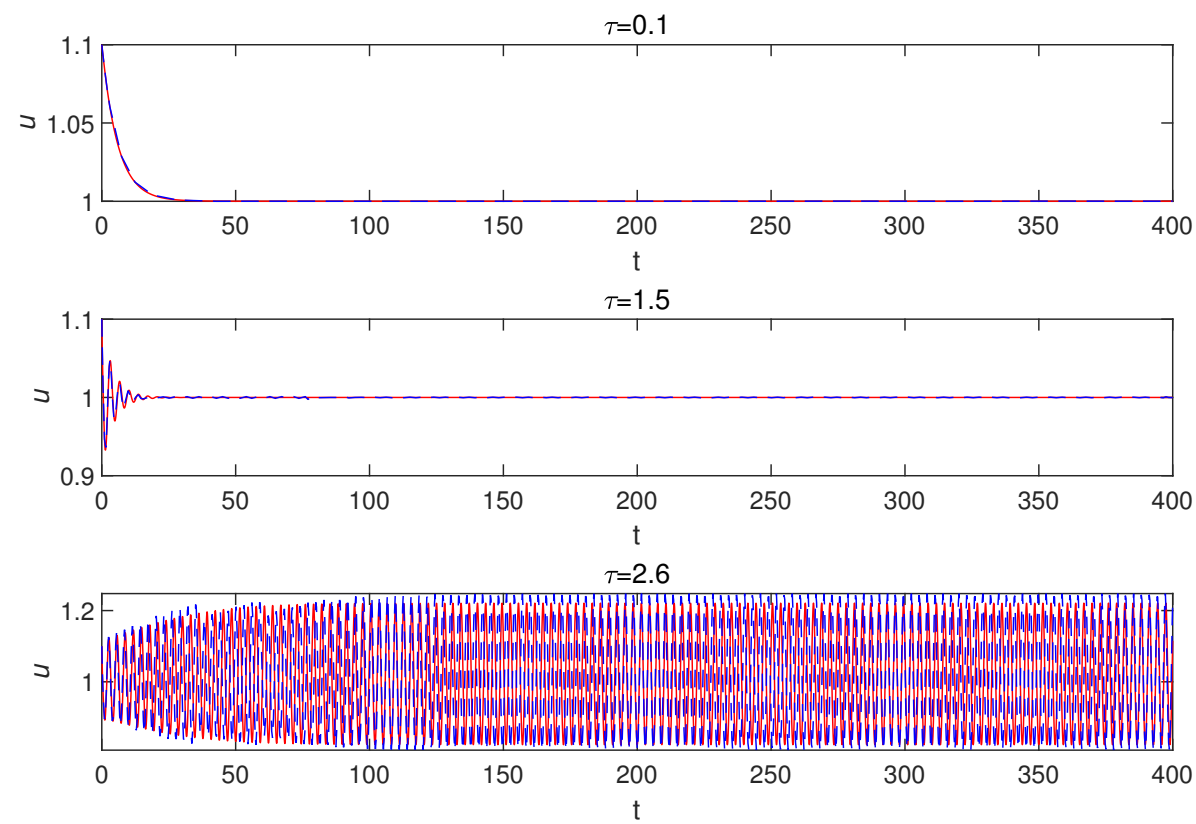

FIG. 1. Analytic solutions (the blue dotted line) and numerical solutions (the red solid line) of (3) with $h=1 / 16$.
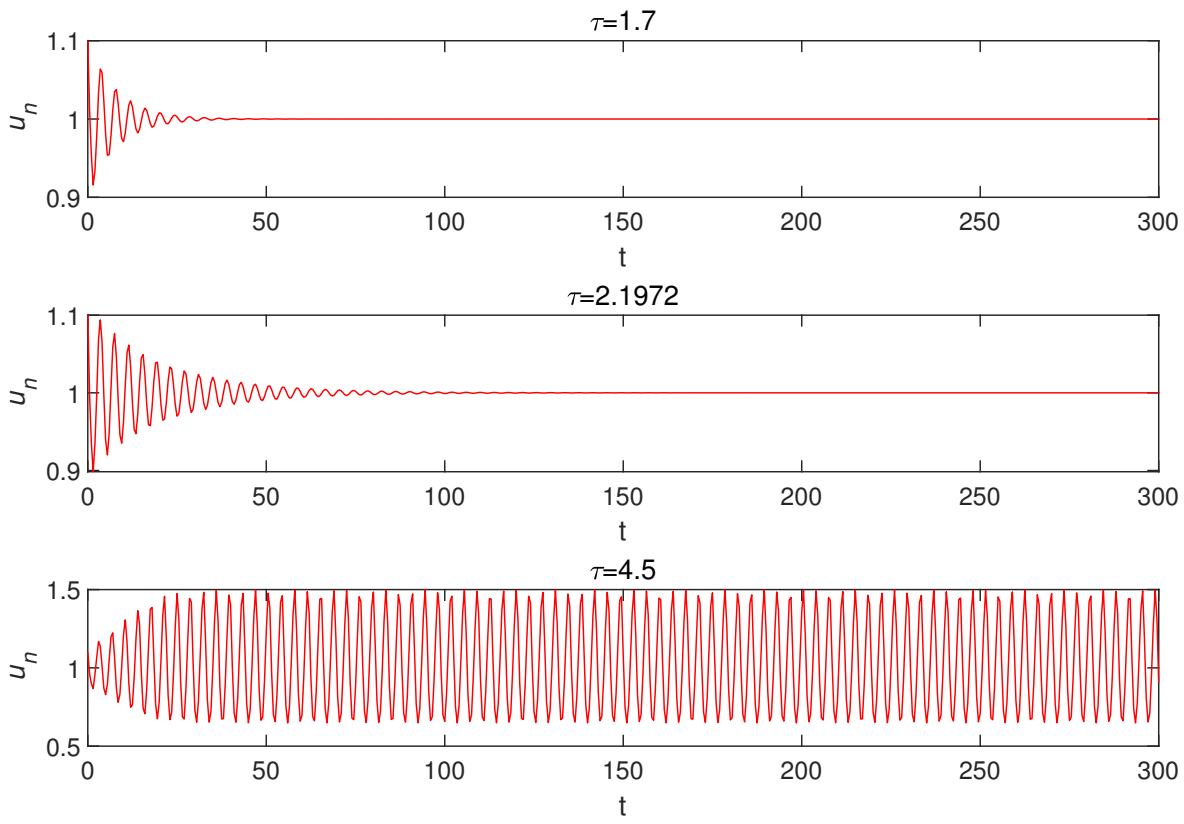

FIG. 2. Numerical solutions of (3) with $h=1 / 2$. 

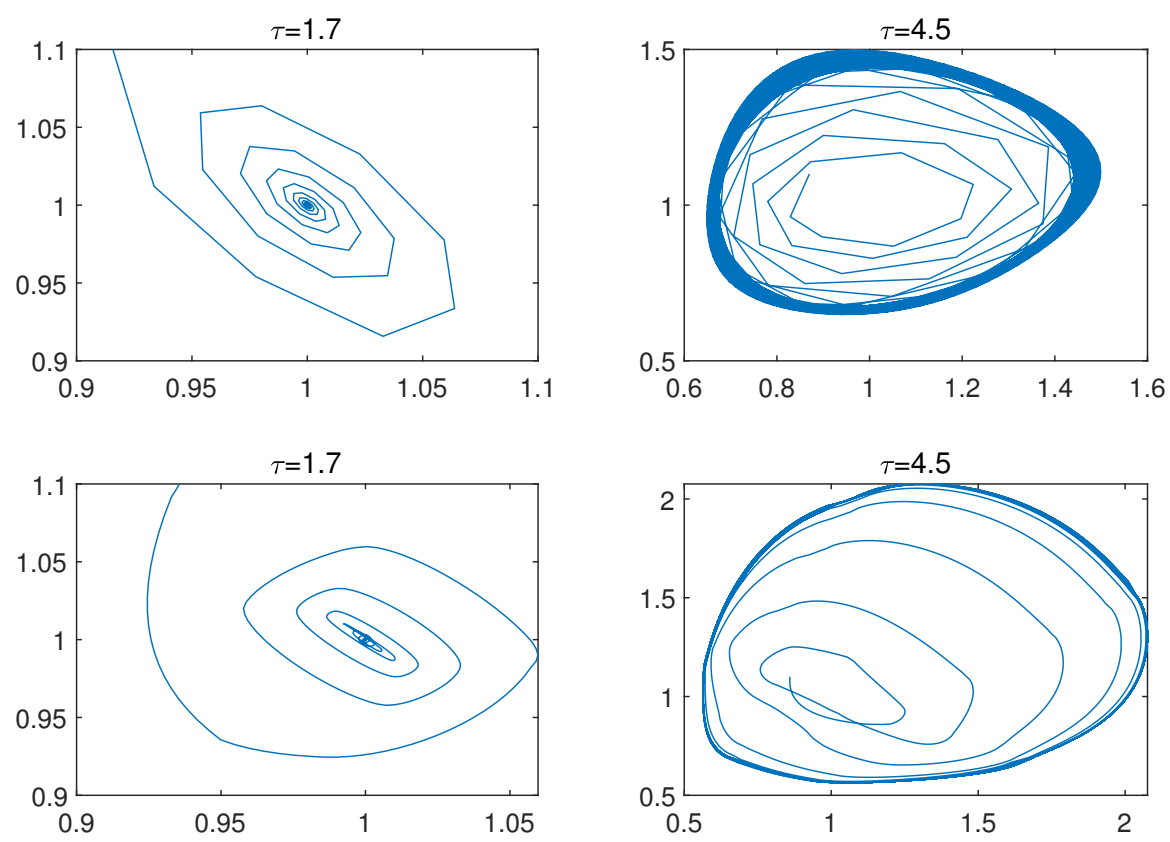

FIG. 3. Phase diagram of (7) with $h=1 / 2$ (upper) and phase diagram of (3) (lower).
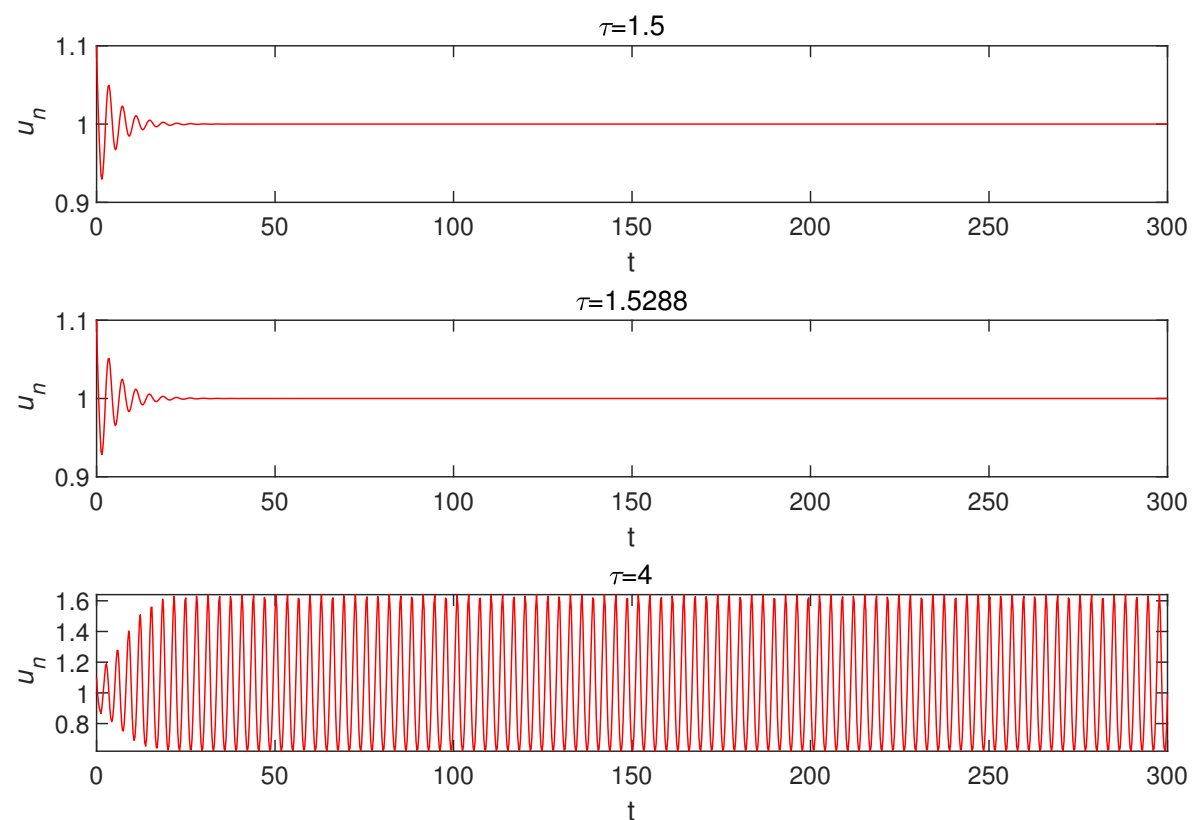

FIG. 4. Numerical solutions of (3) with $h=1 / 4$. 

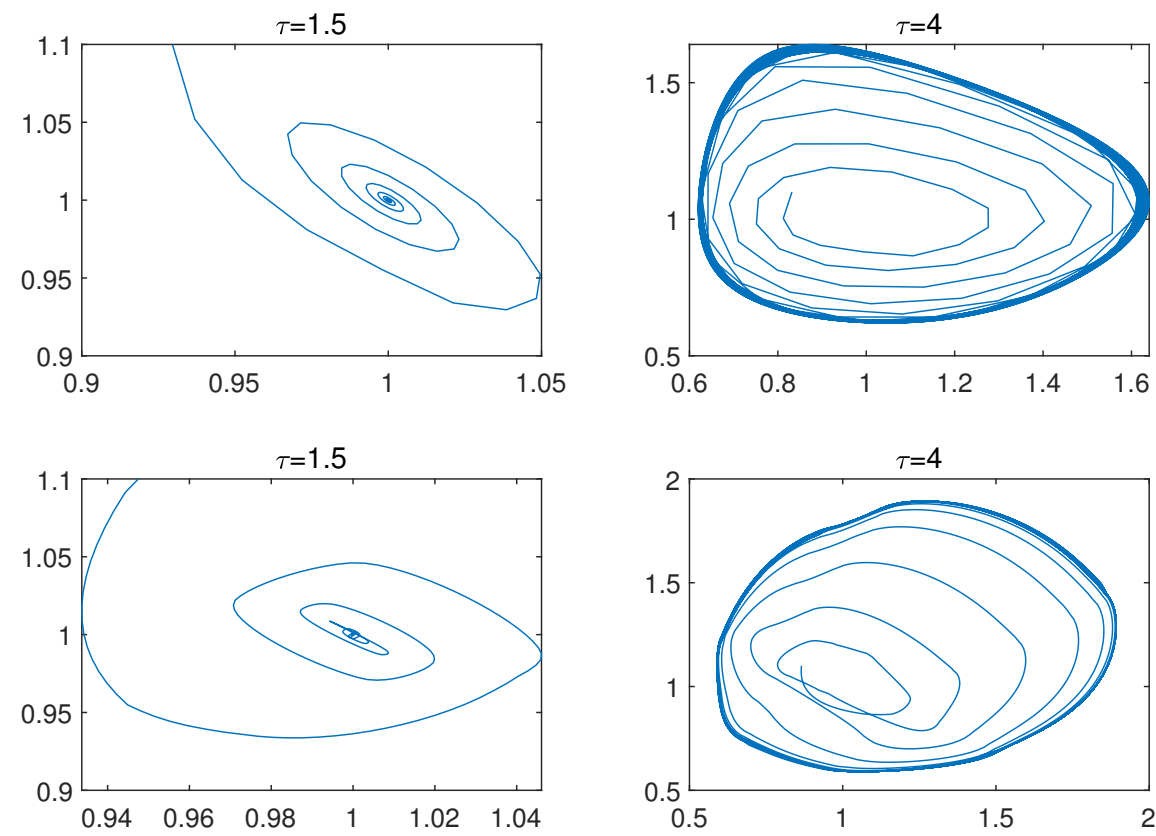

FIG. 5. Phase diagram of (7) with $h=1 / 4$ (upper) and phase diagram of (3) (lower)).
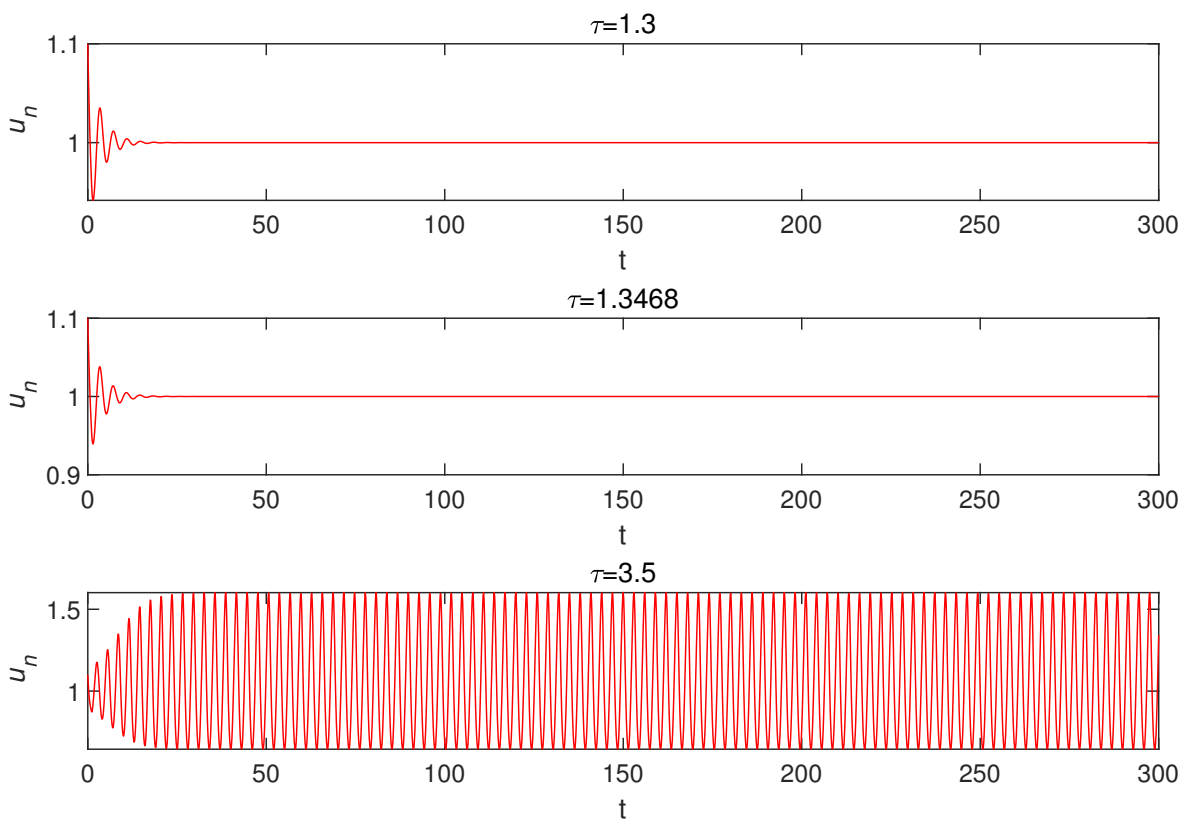

FIG. 6. Numerical solutions of (3) with $h=1 / 8$. 

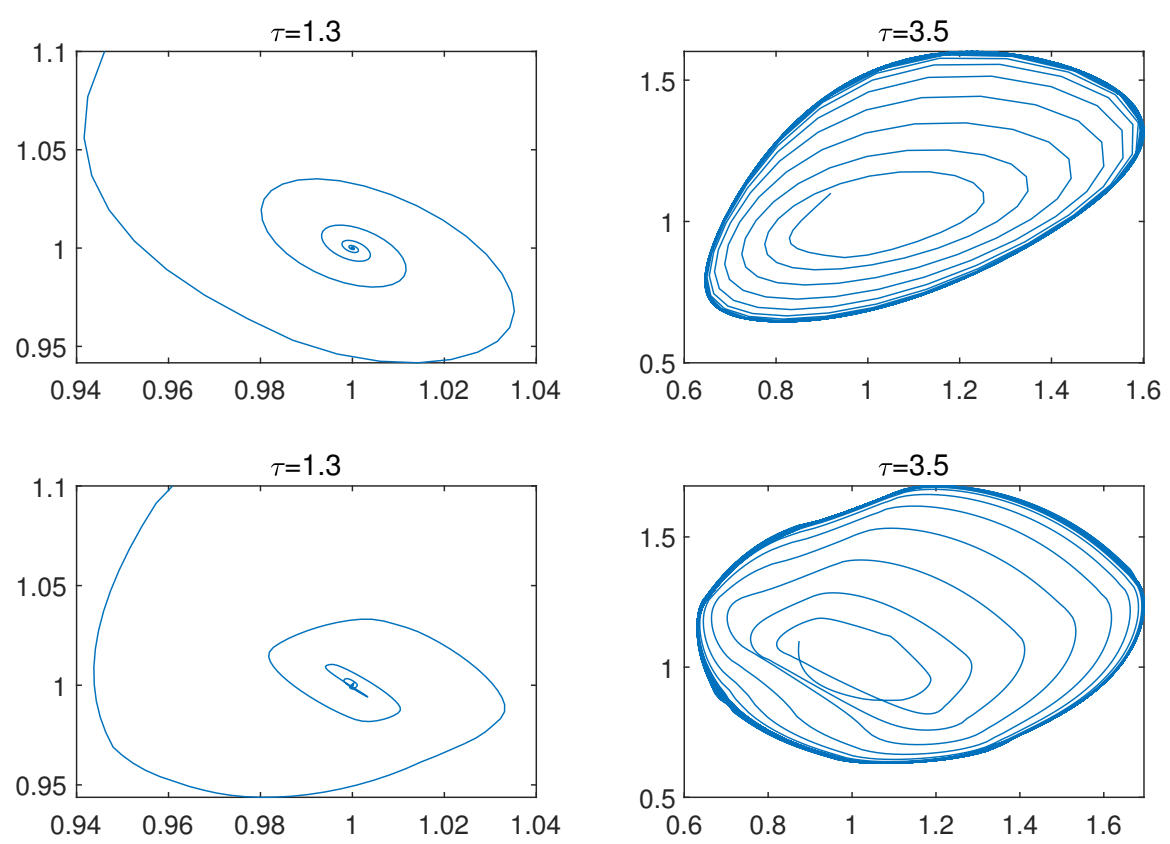

FIG. 7. Phase diagram of (7) with $h=1 / 8$ (upper) and phase diagram of (3) (lower).
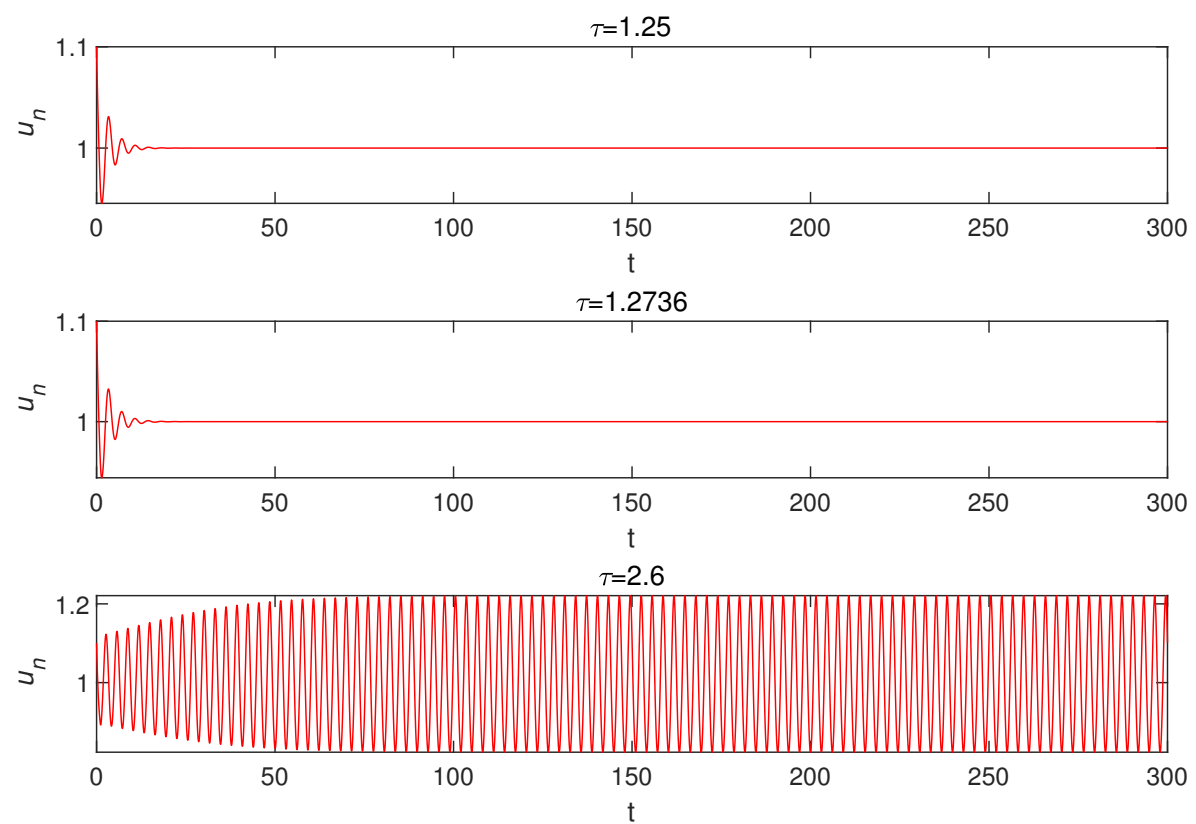

FIG. 8. Numerical solutions of (3) with $h=1 / 16$. 

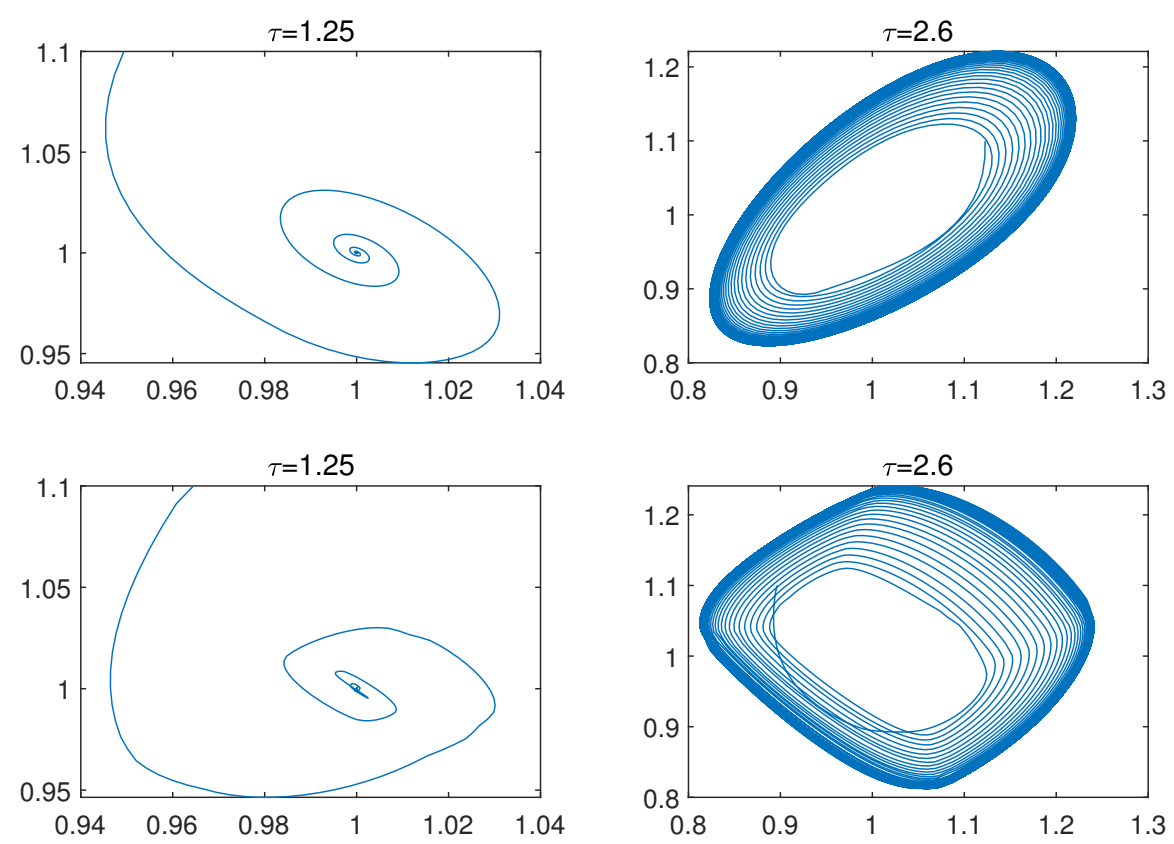

FIG. 9. Phase diagram of (7) with $h=1 / 16$ (upper) and phase diagram of (3) (lower). 